\title{
WEAK IDEAL INVARIANCE AND ORDERS IN ARTINIAN RINGS
}

\author{
JOHN A. BEACHY
}

\begin{abstract}
It is known that a ring $R$ with Krull dimension is an order in an Artinian ring if $R$ is $K$-homogeneous and the prime radical $N$ of $R$ is weakly ideal invariant. The notion of weak ideal invariance can be interpreted in torsion theoretic terms, yielding a shorter and more conceptual proof of this result. In addition, it is shown that the orders in Artinian rings which arise in this fashion are precisely those for which $R / N$ is $K$-homogeneous.
\end{abstract}

Gordon showed in [5] that if $R$ is a fully bounded Noetherian ring having Krull dimension $\alpha$, then $R$ is an order in an Artinian ring if every nonzero left ideal of $R$ has Krull dimension $\alpha$. (If $|Y|$ denotes the Krull dimension of the module ${ }_{R} Y$, then $Y$ is said to be $K$-homogeneous if $|X|=|Y|$ for every nonzero submodule $X$ of $Y$.) This result was extended by Krause, Lenagan and Stafford in [8] to the class of rings with Krull dimension on the left whose prime radical is weakly ideal invariant. (An ideal $I$ of $R$ is said to be (left) weakly ideal invariant if $|I / I D|<|R / I|$ for any left ideal $D$ of $R$ with $|R / D|<|R / I|$.) The purpose of this note is to give a short, direct proof of the latter result, by using torsion theoretic techniques to extend several known results from the class of left Noetherian rings to the class of rings with Krull dimension.

The reader is referred to [6] for the basic facts concerning rings

Received 20 October 1980. 
with Krull dimension, and to [12] for the relevant facts about torsion radicals (that is, left exact radicals). Gordon's proof of the main theorem in [5] utilized the torsion radical $\tau_{\alpha}$, where $\tau_{\alpha}$ is defined on the module $R^{X}$ by setting $\tau_{\alpha}(X)=\{x \in X|| R x \mid<\alpha\}$, where $\alpha=|R|$. Mïl ler showed [10, Theorem 6] that for a left Noetherian ring $R, \tau_{\alpha}$ is determined by the ideal $N_{\alpha}$ defined as the intersection of all prime ideals $P$ of $R$ for which $|R / P|=\alpha$.

The connection with weak ideal invariance appears to be best seen by considering certain general results on torsion radicals. Recall that for a torsion radical $\tau$, an $R$-submodule $X \subseteq Y$ is said to be $\tau$-dense if $Y / X$ is $\tau$-torsion (that is, $\tau(Y / X)=Y / X$ ) and $\tau$-closed if $Y / X$ is $\tau$-torsionfree (that is, $\tau(Y / X)=(0)$ ). Jategaonkar defined the following notion in [7]: an ideal $I$ "deflates" the torsion radical $\tau$ if $I D$ is $\tau$-dense in $I$, for all $\tau$-dense left ideals $D$ of $R$. Applying this definition to the ideal $N_{\alpha}$ defined above, it can be seen that $N_{\alpha}$ is weakly ideal invariant if and only if $N_{\alpha}$ deflates $\tau_{\alpha}$. The proof of the main theorem then involves a theorem of Cozzens and Sandomierski [4, Theorem 2.3], which states that for a semiprime Goldie ideal $I$, the set $C(I)$ of elements regular modulo $I$ is a left ore set if and only if $I$ deflates the torsion radical defined by $C(I)$, and every left ideal of $R$ which is not $\tau$-dense is contained in a maximal $\tau$-closed left ideal. Combining [2, Proposition 6] and [9, Theorem 1.4] shows that in the quotient category determined by $\tau_{\alpha}$, the localization of $R$ has finite length. Thus if $R$ is K-homogeneous and $N_{\alpha}$ is weakly ideal invariant, then $R$, is a left order in the localization at $N_{\alpha}$, which is a left Artinian ring.

Throughout the paper, $R$ will be assumed to be an associative ring with identity, and all modules will be assumed to be unital left $R$-modules. The intersection of all prime ideals of $R$, which will be referred to as the prime radical of $R$, will be denoted by $N$. It will generally be assumed that $R$ has: Krull dimension on left ideals. Recall that the Krull dimension of a module $R^{X}$ is defined inductively as follows: $|x|=-1$ if $x=(0) ;|x|=0$ if $x$ is Artinian; if $\alpha$ is 
an ordinal and $|x|+\alpha$, then $|x|=\alpha$ if there is no infinite descending chain $x=x_{0} \supset x_{1} \supset \ldots$ of submodules such that $\left|x_{i-1} / x_{i}\right| \nless \alpha$ for all $i=1,2, \ldots$.

It follows from the assumption that $R$ has Krull dimension that the prime radical $N$ is nilpotent. Furthermore, if $I$ is any semiprime ideal of $R$, then $I$ is a Goldie ideal, that is, $R / I$ is a left order in a semisimple Artinian ring. General results on localization at semiprime Goldie ideals (see $[1 ; \S 3]$ ) can thus be applied to semiprime ideals of $R$. If $I$ is an ideal, then the set $C(I)$ of elements regular modulo $I$ is defined by

$$
C(I)=\{c \in R \mid \text { for all } r \in R \text {, cr } \in I \text { or } r c \in I \text { implies } r \in I\} \text {. }
$$

If $I$ is a semiprime Goldie ideal, then the set $C(I)$ determines a torsion radical $\tau$ by defining, for any module $R^{X}$,

$\tau(X)=\{x \in X \mid$ for all $r \in R$ there exists $c \in C(I)$ with $\operatorname{crx}=0\}$.

It has been shown that $\tau$ is cogenerated by the injective envelope $E(R / I)$ of the $R$-module $R / I$, so that $\tau(X)$ is the intersection of all kernels of $R$-homomorphisms $f: X \rightarrow E(R / I)$. Note that if the left ideal $D$ is $\tau$-dense in $R$, then $D$ contains an element $c \in C(I)$.

The first lemma gives necessary and sufficient conditions under which an ideal $I$ deflates a torsion radical $T$. The second condition is the one originally given by Jategaonkar [7]. If $X$ is an $R$-submodule of $Y$, then the $\tau$-closure of $X$ in $Y$ is the intersection of all $\tau$-closed submodules of $Y$ which contain $X$, and it will be denoted by $c l_{\tau}(X)$.

LEMMA 1. Let $I$ be an ideal of $R$, and let $\tau$ be a torsion radical. Then the following conditions are equivalent:

(1) if $D$ is a $\tau$-dense left ideal of $R$, then ID is $\tau$-dense in $I$;

(2) if $A$ is any left ideal of $R$, then $I A$ is $\tau$-dense in $I \cdot \mathrm{cl}_{\tau}(A)$;

(3) if $C$ is a $\tau$-closed left ideal of $R$, then $I^{-1} C=\left\{r \in R \mid I_{r} \subseteq C\right\}$ is t-closed in $R$. 
Proof. (1) implies (3). Let $C$ be a $\tau$-closed left ideal of $R$. To show that $I^{-1} C$ is $\tau$-closed in $R$, it suffices to show that if $x \in R$ and $D x \subseteq I^{-1} C$ for a $\tau$-dense left ideal $D$, then $x \in I^{-1} C$. Suppose $D x \subseteq I^{-1} C$, where $D$ is $\tau$-dense. Since $I D$ is by assumption $\tau$-dense in $I$, for each $a \in I$ there exists a $\tau$-dense left ideal $D^{\prime}$ with $D^{\prime} a \subseteq I D$. Then $D^{\prime} a x \subseteq I D x \subseteq C$ implies that $a x \in C$, since $C$ is T-closed. This shows that $x \in I^{-1} C$, and so $I^{-1} C$ is $\tau$-closed.

(3) implies (2). For any left ideal $A$, let $C=c l_{\tau}(I A)$. Then $C$ is $\tau$-closed, and so by assumption $I^{-1} C$ is $\tau$-closed. Now $I A \subseteq \mathrm{cl}_{\tau}(I A)$, so $A \subseteq I^{-1} C$, and therefore $\mathrm{cl}_{\tau}(A) \subseteq I^{-1} C$. Thus $I \cdot c{ }_{\tau}(A) \subseteq{ }^{c l}{ }_{\tau}(I A)$, which shows that $I A$ is $\tau$-dense in $I \cdot c I_{\tau}(A)$.

(2) implies (1). This follows immediately from the fact that if $D$ is a $\tau$-dense left ideal, then $c I_{\tau}(D)=R$.

Let $I$ be an ideal of $R$, and let $D$ be a $t$-dense left ideal. Note that in general, to show that $I D$ is $\tau$-dense in $I$, it suffices to show that $I D$ is $\tau$-dense in $I \cap D$. This follows from the observation that $I \cap D$ is always $\tau$-dense in $I$, since $I /(I \cap D) \simeq(I+D) / D$ and submodules of $\tau$-torsion modules are $\tau$-torsion. The next lemma extends part of Theorem 2.3 of [4], where $I$ is assumed to be a semiprime Goldie ideal and $\tau$ is the torsion radical defined by $C(I)$.

LEMMA 2. Let $I$ be an ideal of $R$ and let $\tau$ be a torsion radical. If the filter of $\tau$-dense left ideals has a basis of principal left ideals $\{R s \mid s \in S\}$ such that each $s \in S$ is a regular element moduzo $I$, then $I$ deflates $\tau$.

Proof. Given a $\tau$-dense left ideal $D$ and $x \in I \cap D$, there exist principal $\tau$-dense left ideals $R s$ and $R t$, with $s, t \in S$, such that $R s \subseteq D$ and $R t x \subseteq R s$. Thus $t x=a s$ for some $a \in R$, and then as $=t x \in I$ implies that $a \in I$, since $s$ is a regular element modulo $I$. It follows that $I D$ is $\tau$-dense in $I \cap D$, since Rtx $=$ Ras $\subseteq I D$.

LEMMA 3. Let $I$ be an ideal of $R$ and let $\sigma$ and $\tau$ be torsion radicals. Assume that $\tau \leq \sigma$ and that $\tau=\sigma$ on any $R / I$-module. If $I$ 
deflates $\sigma$, then $I$ deflates $\tau$.

Proof. Let $D$ be a $\tau$-dense left ideal. The assumption that $\tau \leq \sigma$ is equivalent to the condition that every $\tau$-dense left ideal is $\sigma$-dense, so $D$ is $\sigma$-dense. If $I$ deflates $\sigma$, then $(I \cap D) / I D$ is $\sigma$-torsion, so by assumption it must be $\tau$-torsion, since $(I \cap D) / I D$ is an $R / I$-module. This shows that $I$ deflates $\tau$.

The next theorem was proved by Brown, Lenagan and Stafford for left Noetherian rings [3, Theorem 3.1]. Their proof utilizes techniques which are quite different, and requires several results from [8].

THEOREM 4. Let $R$ be a ring with Krulz dimension on the left, and let $I$ be a semiprime ideal of $R$. If $R / I$ is K-homogeneous and $C(I)$ is a left ore set, then $I$ is weakly ideal invariant.

Proof. Let $\sigma$ be the torsion radical defined by $C(I)$, let $|R / I|=\alpha$, and let $\tau_{\alpha}$ be the torsion radical determined by modules of Krull dimension less than $\alpha$, that is, a left ideal $D$ is $\tau_{\alpha}$-dense if $|R / D|<\alpha$. If $C(I)$ is a left Ore set, then $\{R c \mid c \in C(I)\}$ is a basis for the filter of $\sigma$-dense left ideals, so $I$ deflates $\sigma$ by Lemma 2 . If $D$ is a $\tau_{\alpha}$-dense left ideal, then $\operatorname{Hom}(R / D, E(R / I))=(0)$ since $|R / D|<\alpha$ and by assumption every $R$-submodule of $R / I$ has Krull dimension equal to $\alpha$, so $D$ is $\sigma$-dense. If $D \supseteq I$ is a $\sigma$-dense left ideal, then $D$ contains an element $c \in C(I)$. Since $R \supset R c+I \supset R c^{2}+I \supset \ldots$ is an infinite descending chain, it follows that $|R /(R c+I)|<|R / I|=\alpha$, so $|R / D| \leq|R /(R c+I)|<\alpha$ and $D$ is $\tau_{\alpha}$-dense. Therefore $\sigma=\tau_{\alpha}$ for any $R / I$-module. Thus the conditions of Lemma 3 are satisfied and $I$ deflates $\tau_{\alpha}$, which is equivalent to the statement that $I$ is weakly ideal invariant.

THEOREM 5. Let $R$ be a ring with Krull dimension a on the left. Let $N_{\alpha}$ be the intersection of all prime ideals $P$ of $R$ such that $|R / P|=\alpha$, and let $\tau$ be the torsion radical determined by $C\left(N_{\alpha}\right)$. Then a left ideal $D$ is $\tau$-dense if and only if $|R / D|<\alpha$.

Proof. If $P$ is a prime ideal with $|R / P|=\alpha$, then $P$ must be a minimal prime ideal by [6, Corollary 7.2], so $N_{\alpha}$ is a finite intersection 
of prime ideals $\left\{P_{i}\right\}_{i=1}^{n}$ since $R$ has only finitely many minimal prime ideals. Thus $E\left(R / N_{\alpha}\right) \simeq \bigoplus_{i=1}^{n} E\left(R / P_{i}\right)$, and it follows that $R / N_{\alpha}$ is $K$-homogeneous and $\operatorname{Hom}_{R}\left(R / D, E\left(R / N_{\alpha}\right)\right)=(0)$ for any left ideal $D$ with $|R / D|<\alpha$. This shows that if $|R / D|<\alpha$, then $D$ is $\tau$-dense.

Conversely, let $D$ be a $\tau$-dense left ideal. Since the prime radical of $R$ is nilpotent, it follows that $D$ contains a finite product of minimal prime ideals, and so it is possible to construct a finite chain of left ideals $D=D_{0} \subseteq D_{1} \subseteq \ldots \subseteq D_{n}=R$ for which each factor is annihilated by a minimal prime ideal. If $P_{k} D_{k} \subseteq D_{k-1}$ for a minimal prime $P_{k}$ with $\left|R / P_{k}\right|<\alpha$, then $\left|D_{k} / D_{k-1}\right|<\alpha$. On the other hand, if $\left|R / P_{k}\right|=\alpha$, then since $D_{k-1}$ is $\tau$-dense in $D_{k}$, for each $x \in D_{k}$ there exists $c \in C\left(N_{\alpha}\right)$ with $c x \in D_{k-1}$. Since $c \in C\left(P_{k}\right)$ as well, $\left|R+\left(R c / P_{k}\right)\right|<\left|R / P_{k}\right|$ by the standard argument used in Theorem 4. Then $\left(R x+D_{k-1}\right) / D_{k-1}$ is a homomorphic image of $R /\left(R c+P_{k}\right)$, so $\left|\left(R x+D_{k-1}\right) / D_{k-1}\right| \leq\left|R /\left(R c+P_{k}\right)\right|<\alpha$, which shows that $\left|D_{k} / D_{k-1}\right|<\alpha$. It follows that $|R / D|=\max _{k=1, \ldots, n}\left\{\left|D_{k} / D_{k-1}\right|\right\}<\alpha$.

Theorem 5 has been proved for left Noetherian rings by Miller [10, Theorem 6]. It shows that for a ring $R$ with $|R|=\alpha$, the torsion radical $\tau_{\alpha}$ generated by modules of Krull dimension less than $\alpha$ coincides with the torsion radical defined by $C\left(N_{\alpha}\right)$.

THEOREM 6. Let $R$ be a ring with Krull dimension $\alpha$ on the left. Let $N_{\alpha}$ be the intersection of all minimal prime ideals $P$ such that $|R / P|=\alpha$. Then $N_{\alpha}$ is weakly ideal invariant if and only if $c\left(N_{\alpha}\right)$ is a left Ore set.

Proof. If $C\left(N_{\alpha}\right)$ is a left Ore set, then Theorem 4 shows that $N_{\alpha}$ is weakly ideal invariant.

Conversely, suppose that $N_{\alpha}$ is weakly ideal invariant. Proposition 
6 of [2] implies that for any minimal prime ideal $P$, the ring $R$ has the descending chain condition on left ideals closed with respect to the torsion radical defined by $C(P)$. Since $N_{\alpha}$ is a finite intersection of minimal prime ideals, it follows that $R$ has the descending chain condition on $\tau_{\alpha}$-closed left ideals, where $\tau_{\alpha}$ is the torsion radical defined by $C\left(N_{\alpha}\right)$. By the Teply and Miller Theorem [9, Theorem 1.4], $R$ must have the ascending chain condition on $\tau_{\alpha}$-closed left ideals.

Let $c \in C\left(N_{\alpha}\right)$. If $R c$ is not $\tau_{\alpha}$-dense, then by the preceding remarks $R c$ is contained in a proper left ideal $C$ maximal in the set of $\tau_{\alpha}$-closed left ideals. Since $N_{\alpha}$ is by assumption weakly ideal invariant, the conditions of Lemma 1 are satisfied since by Theorem 5 a left ideal $D$ is $\tau_{\alpha}$-dense if and only if $|R / D|<\alpha$. Thus $N_{\alpha}^{-1} C=\left\{r \in R \mid N_{\alpha} r \subseteq C\right\}$ is $\tau_{\alpha}$-closed in $R$, which forces $N_{\alpha}^{-1} C=R$ since $N_{\alpha}^{-1} C \supsetneqq C$. (To see that $N_{\alpha}^{-1} C \neq C$, since $C$ is $\tau_{\alpha}$-closed there exists an $R$-homomorphism $0 \neq f: R / C+E\left(R / N_{\alpha}\right)$, and ker $f=(0)$ since ker $f$ is $\tau_{\alpha}$-closed in $R / C$ and $C$ is a maximal $\tau_{\alpha}$-closed left ideal. Then $X=f(R / C) \cap\left(R / N_{\alpha}\right) \neq(0)$, and $N_{\alpha}^{-1} C$ contains the inverse image in $R$ of any element in $X$. ) Since $N_{\alpha}^{-1} C=R$, it follows that $N_{\alpha} \subseteq C$, so $R c+N_{\alpha} \subseteq C$. This contradicts the fact that $R c+N_{\alpha}$ is $\tau_{\alpha}$-dense in $R$.

The left Ore condition then follows from the fact that if $c \in C\left(N_{\alpha}\right)$, then $R c$ is $\tau_{\alpha}$-dense. In fact, given $c \in C\left(N_{\alpha}\right)$ and $r \in R$, since $R c$ is $\tau_{\alpha}$-dense there exists a $\tau_{\alpha}$-dense left ideal $D$ such that $D r \subseteq R c$, so there exist $c^{\prime} \in D \cap C\left(N_{\alpha}\right)$ and $r^{\prime} \in R$ with $c^{\prime} r=r^{\prime} c$.

The major part of the final theorem is due to Krause, Lenagan and Stafford, who showed in [8, Theorem 8] that a K-homogeneous ring (with Krull dintension) is an order in an Artinian ring if its prime radical is weakly ideal invariant. It should also be noted that Gordon showed in [5, 
Corollary 3] that in a $K$-homogeneous FBN ring, the ring modulo its prime radical is $K$-homogeneous.

THEOREM 7. Let $R$ be a ring with Krull dimension on the left, and let $N$ be the prime radical of $R$. Then the following conditions are equivalent:

(1) $R$ is K-homogeneous and $N$ is weakly ideal invariant;

(2) $R$ is a left order in a left Artinian ring and $R / N$ is $K$-homogeneous.

Proof. (1) implies (2). Let $|R|=\alpha$, let $N_{\alpha}$ be the intersection of minimal prime ideals $P$ with $|R / P|=\alpha$, and let $\tau_{\alpha}$ be the torsion radical defined by $C\left(N_{\alpha}\right)$. It will first be shown that if $N$ is weakly ideal invariant, then $N_{\alpha}$ is weakly ideal invariant. If $D$ is a left ideal with $|R / D|<\left|R / N_{\alpha}\right|=\alpha$, then by assumption $|N / N D|<\alpha$. Let $I$ be the intersection of minimal prime ideals $P$ of $R$ with $|R / P|<\alpha$. Then $|R / I|<\alpha$ and $I N_{\alpha} \subseteq I \cap N_{\alpha}=N$, so $N_{\alpha} / N$ is an $R / I$-module, which forces $\left|N_{\alpha} / N\right|<\alpha$. Since $N D \subseteq N_{\alpha} D$, it follows that

$$
\left|N_{\alpha} / N_{\alpha} D\right| \leq\left|N_{\alpha} / N D\right|=\max \left\{\left|N_{\alpha} / N\right|,|N / N D|\right\}<\alpha .
$$

As in the proof of Theorem 6, $R$ has ascending chain condition and descending chain condition on $\tau_{\alpha}$-closed left ideals. If $R$ is $K$-homogeneous, then $R$ is $\tau_{\alpha}$-torsionfree, and so any left annihilator of $R$ is $\tau_{\alpha}$-closed. Thus $R$ has ascending chain condition on left annihilators, so $C\left(N_{\alpha}\right)$ is a left denominator set by Theorem 6 and [12, Chapter II, Proposition 1.5]. Localization at $N_{\alpha}$ is thus given by inverting elements of $C\left(N_{\alpha}\right)$, and so $R$ is a left order in a left Artinian ring since the left ideals of the localization correspond to $\tau_{\alpha}$-closed left ideals of $R$.

(2) implies (1). Assume that condition (2) holds. Then Theorem 4 shows that $N$ must be weakly ideal invariant, and $R$ must be $K$-homogeneous since $R / N$ is $K$-homogeneous and $E(R / N)$ is a faithful 
$R$-module.

\section{References}

[1] John A. Beachy, "Some aspects of noncommutative localization", Noncormutative ring theory, 2-31 (Internat. Conf., Kent State University, 1975. Lecture Notes in Mathematics, 545. SpringerVerlag, Berlin, Heidelberg, New York, 1976).

[2] John A. Beachy, "Injective modules with both ascending and descending chain conditions on annihilators", Comm. Algebra 6 (1978), 1777-1788.

[3] K.A. Brown, T.H. Lenagan and J.T. Stafford, "Weak ideal invariance and localisation", J. London Math. Soc. (2) 21 (1980), 53-61.

[4] J.H. Cozzens and F.L. Sandomierski, "Localization at a semiprime ideal of a right Noetherian ring", Comm. Algebra 5 (1977), 707-726.

[5] Robert Gordon, "Artinian quotient rings of FBN rings", J. AZgebra 35 (1975), 304-307.

[6] Robert Gordon and J.C. Robson, Krull dimension (Memoirs of the American Mathematical Society, 133. American Mathematical Society, Providence, Rhode Island, 1973).

[7] Arun Vinayak Jategaonkar, "Relative Krull dimension and prime ideals in right Noetherian rings", Comm. Algebra 2 (1974), 429-468.

[8] Günter Krause, T.H. Lenagan and J.T. Stafford, "Ideal inveriance and Artinian quotient rings", J. Algebra 55 (1978), 145-154.

[9] Robert W. Miller and Mark L. Teply, "The descending chain condition relative to a torsion theory", Pacific J. Math. 83 (1979), 207-219.

[10] Bruno J. Müller, "Ideal invariance and localization", Comm. Algebra 7 (1979), 415-441.

[11] Constantin Năstăsescu, "Conditions de finitude pour les modules", Rev. Roumaine Math. Pures Appl. 24 (1979), 745-758. 
[12] Bo Stenström, Rings of quotients. An introduction to methods of ming theory (Die Grundlehren der mathematischen Wissenschaften, 217. Springer-Verlag, Berlin, Heidelberg, New York, 1975).

Department of Mathematical Sciences, Northern IIlinois Unversity,

DeKalb, IIIinois 60115 , USA. 